\title{
On the blow-up of solutions for the unstable sixth order parabolic equation
}

\author{
Zhenbang Li and Changchun Liu
}




\title{
ON THE BLOW-UP OF SOLUTIONS FOR THE UNSTABLE SIXTH ORDER PARABOLIC EQUATION
}

\author{
ZHENBANG LI AND CHANGCHUN LIU
}

Received 3 May, 2012

\begin{abstract}
We study the universal blow-up of sixth-order parabolic thin film equation with the initial boundary conditions. We prove that the problem in finite time blow-up will happen, if the initial datum $u_{0} \in C^{6+\alpha}(\bar{\Omega})$ with $-\int_{\Omega}\left(H\left(u_{0}\right)+\frac{1}{2}\left|\Delta u_{0}\right|^{2}\right) d x \geq 0$. And then, we get some nondegeneracy results on blow-up for this problem.
\end{abstract}

2000 Mathematics Subject Classification: 35K55; 35K90; 76A20

Keywords: blow-up, nondegeneracy, sixth order parabolic equation

\section{INTRODUCTION}

In this paper, we consider the following initial boundary problem of sixth-order equation

$$
\left\{\begin{aligned}
u_{t}-\Delta\left(\Delta^{2} u-|u|^{p-1} u\right) & =0, & & \text { in } \Omega \times(0, T), \\
u=\Delta u=\Delta^{2} u & =0, & & \text { on } \partial \Omega \times[0, T), \\
u & =u_{0}, & & \text { in } \Omega \times\{0\},
\end{aligned}\right.
$$

where $\Omega \subset R^{N}$ is a bounded smooth domain, $p>1$.

During the past years, only a few works have been devoted to the sixth-order parabolic equation $[1,4,5,7]$.

Recently, Evans, Galaktionov and King [4,5] considered the sixth-order thin film equation containing an unstable (backward parabolic) second-order term

$$
\frac{\partial u}{\partial t}=\operatorname{div}\left[|u|^{n} \nabla \Delta^{2} u\right]-\Delta\left(|u|^{p-1} u\right), n>0, p>1 .
$$

By a formal matched expansion technique, they show that, for the first critical exponent $p=p_{0}=n+1+\frac{4}{N}$ for $n \in\left(0, \frac{5}{4}\right)$, where $N$ is the space dimension, the free-boundary problem with zero-height, zero-contact-angle, zero-moment, and zeroflux conditions at the interface admits a countable set of continuous branches of radially symmetric self-similar blow-up solutions $u_{k}(x, t)=(T-t)^{-\frac{N}{n N+6}} f_{k}(y)$, $y=\frac{x}{(T-t)^{\frac{1}{n N+6}}}$, where $T>0$ is the blow-up time. 
In fact, when $n=0$, the equation (1.1) is obtained. In this paper we study the universal blow-up and some nondegeneracy results on blow-up of the equation (1.1). Our method about universal finite time blow-up is similar to that of Elliott and Zheng [3] which treats the blow-up problem for Cahn-Hilliard equation. We can show that if the initial datum $u_{0} \in C^{6+\alpha}(\bar{\Omega})$ with $-\int_{\Omega}\left(H\left(u_{0}\right)+\frac{1}{2}\left|\Delta u_{0}\right|^{2}\right) d x \geq 0$, then the solution to the above problem (1.1) should blow up in finite time.

We also establish some nondegeneracy results on the blow-up of the problem. We mainly follow the purpose of Giga and Kohn [6] and Cheng and Zheng [2]. More accurately, there is a constant $\varepsilon>0$, depending on $n, p$ and the constant in the estimates of the fundamental solution to $u_{t}-\Delta^{3} u=0$ (see (3.1) below), such that if $u$ is a solution of the equation

$$
u_{t}-\Delta\left(\Delta^{2} u-|u|^{p-1} u\right)=0, \quad \text { on } \quad Q_{r}=B_{r}(a) \times\left[t_{1}-r^{6}, t_{1}\right),
$$

where $1<p<3, a \in \mathbb{R}^{n}, t_{1} \in \mathbb{R}$ and $0<r \leq 1$, and if

$$
|u(x, t)| \leq \varepsilon\left(t_{1}-t\right)^{-\frac{2}{3(p-1)}} \quad \text { for } \quad \text { all } \quad(x, t) \in Q_{r},
$$

then $u$ does not blow up at $\left(a, t_{1}\right)$.

The following sections include our main results. In Section 2, we establish universal finite time blow-up. Section 3 is devoted to the nondegeneracy results on the blow-up.

\section{UNIVERSAL FINITE TIME BLOW-UP}

Theorem 1. Assume $u_{0} \in C^{6+\alpha}(\bar{\Omega})$ with $-\int_{\Omega}\left(H\left(u_{0}\right)+\frac{1}{2}\left|\Delta u_{0}\right|^{2}\right) d x \geq 0$. Then the solution of the problem (1.1) must blow up at a finite time, namely, for some $T>0$

$$
\lim _{t \rightarrow T}\|u(t)\|=+\infty
$$

where $H(u)=-\frac{|u|^{p+1}}{p+1}$.

Proof. Let

$$
F(t)=\int_{\Omega}\left(H(u)+\frac{1}{2}|\Delta u|^{2}\right) d x,
$$

then

$$
\begin{aligned}
\frac{d F(t)}{d t} & =\int_{\Omega}\left(-|u|^{p-1} u \varphi(u) u_{t}+\frac{1}{2} \Delta u \Delta u_{t}\right) d x \\
& =\int_{\Omega}\left(-|u|^{p-1} u+\frac{1}{2} \Delta^{2} u\right) u_{t} d x \\
& =-\int_{\Omega}\left|\nabla\left(-|u|^{p-1} u+\frac{1}{2} \Delta^{2} u\right)\right|^{2} d x \leq 0 .
\end{aligned}
$$

So

$$
2 \int_{\Omega} H(u) d x-2 F(0) \leq-\|\Delta u\|^{2},
$$


where

$$
F(0)=\int_{\Omega}\left(H\left(u_{0}\right)+\frac{1}{2}\left|\Delta u_{0}\right|^{2}\right) d x .
$$

Let $\phi$ be the unique solution to

$$
\begin{cases}\Delta \phi=u, & \text { in } \Omega, \\ \nabla \phi=0, & \text { on } \partial \Omega .\end{cases}
$$

It is easy to get that

$$
\|\nabla \phi\|^{2} \leq C\|\Delta \phi\|_{2}^{2} \leq C\|u\|^{2} .
$$

Now multiplying (1.1) by $\phi$ and integrating with respect $x$, we obtain

$$
\begin{aligned}
\frac{d}{d t}\|\nabla \phi\|^{2} & =-2 \int_{\Omega} \varphi(u) u d x-2\|\Delta u\|^{2} d x \\
& \geq 4 \int_{\Omega} H(u) d x-4 F(0)-2 \int_{\Omega} \varphi(u) u d x \\
& =\int_{\Omega}\left(2-\frac{4}{p+1}\right)|u|^{p+1} d x-4 F(0) \\
& \geq \frac{2(p-1)}{p+1}\left(\int_{\Omega} u^{2} d x\right)^{\frac{p+1}{2}}-4 F(0) .
\end{aligned}
$$

Combining (2.2), (2.3) and $-F(0) \geq 0$, we have

$$
\frac{d}{d t}\|\nabla \phi\|^{2} \geq \frac{2 C(p-1)}{p+1}\|\nabla \phi\|^{p+1} .
$$

Let $y(t)=\|\nabla \phi\|_{2}^{2}$ with $t \in[0, T)$, then

$$
y^{\prime}(t) \geq \gamma(y(t))^{\frac{p+1}{2}},
$$

where $\gamma=\frac{2 C(p-1)}{p+1}$. A direct integration of (2.5) then yields

$$
y^{\frac{p-1}{2}}(t) \geq \frac{1}{y^{\frac{1-p}{2}}(0)-\frac{p-1}{2} \gamma t} .
$$

It turns out that the solution of the problem (1.1) will blow up in finite time. The proof of this theorem is completed.

\section{NONDEGENERACY RESULTS ON THE BLOW-UP}

Let $\Gamma(x, t)$ be the fundamental solution to $u_{t}-\Delta^{3} u=0$. According to [8], we have the follow inequalities:

$$
\left|D_{t}^{\mu} D_{x}^{v} \Gamma(x, t)\right| \leq C t^{-\frac{1}{6}(n+6 \mu+v)} \exp \left\{-\omega \frac{|x|^{\frac{6}{5}}}{t^{\frac{1}{5}}}\right\}, \quad t>0,
$$

where $C>0, \omega>0$ are constants, and $\mu, v$ are nonnegative integers. 
Our purpose in this section is to have some nondegeneracy results on the blow-up. We state that the solution $u(x, t)$ to blows up at $\left(a, t_{1}\right)$ if it is not locally bounded nearby, i.e., if there is a sequence $\left\{\left(x_{k}, \tau_{k}\right)\right\} \subset \Omega \times\left[0, t_{1}\right)$ with $\left(x_{k}, \tau_{k}\right) \rightarrow\left(a, t_{1}\right)$ as $k \rightarrow \infty$ such that $\left|u\left(x_{k}, \tau_{k}\right)\right| \rightarrow \infty$.

Theorem 2. There is a constant $\varepsilon>0$, depending on $n, p$ and the constant in (3.1), such that if $u$ is a solution of the equation

$$
u_{t}-\Delta\left(\Delta^{2} u-|u|^{p-1} u\right)=0, \quad \text { on } \quad Q_{r}=B_{r}(a) \times\left[t_{1}-r^{6}, t_{1}\right),
$$

where $1<p<3, a \in \mathbb{R}^{n}, t_{1} \in \mathbb{R}$ and $0<r \leq 1$, and if

$$
|u(x, t)| \leq \varepsilon\left(t_{1}-t\right)^{-\frac{2}{3(p-1)}} \quad \text { for } \text { all }(x, t) \in Q_{r},
$$

then $u$ does not blow up at $\left(a, t_{1}\right)$.

Next, we introduce the two lemma which will be used in the article and whose proofs can be found in [2] and [6].

Lemma 1. For $0<a<1, \theta>0$, and $0<h<1$, the integral

$$
I(h)=\int_{h}^{1}(s-h)^{-a} s^{-\theta} d s,
$$

satisfies

$$
\begin{aligned}
& \text { (1) } I(h) \leq\left(\frac{1}{1-a}+\frac{1}{a+\theta-1}\right) \text { if } a+\theta>1 \text {, } \\
& \text { (2) } I(h) \leq \frac{1}{1-a}+|\log h| \text { if } a+\theta=1, \\
& \text { (3) } I(h) \leq \frac{1}{1-a-\theta} \text { if } a+\theta<1 .
\end{aligned}
$$

Lemma 2. If $y(t), r(t)$ and $q(t)$ are continuous functions defined on $\left[t_{0}, t_{1}\right]$, such that $y(t) \leq y_{0}+\int_{t_{0}}^{t} y(s) r(s) d s+\int_{t_{0}}^{t} q(s) d s, t_{0} \leq t \leq t_{1}$, and $r(t) \geq 0$ on $\left[t_{0}, t_{1}\right]$, then

$$
y(t) \leq \exp \left\{\int_{t_{0}}^{t} r(\tau) d \tau\right\}\left[y_{0}+\int_{t_{0}}^{t} q(\tau) \exp \left\{-\int_{t_{0}}^{t} r(\sigma) d \sigma\right\} d \tau\right] .
$$

Then, we began to prove the main Theorem 2 .

Proof. Without loss of generality, we may assume $a=0$ and $t_{1}=0$. By scaling, it is sufficient to consider the case $r=1$. In the fact, if $u$ satisfies the assumptions of the theorem with $r<1$, then $u_{r}(x, t)=r^{\frac{4}{p-1}} u\left(r x, r^{6} t\right)$ satisfies them with $r=1$ (using the same $\varepsilon$ ), and clearly $u_{r}$ blow up at $(0,0)$ if $u$ does.

Let $\phi$ be a smooth function supported on $B_{1}(0)$ such that $\phi \equiv 1$ on $B_{\frac{1}{2}}(0)$ and $0 \leq \phi \leq 1$. Consider $\omega=\phi u$; then $\omega_{t}-\Delta^{3} \omega==g$ where 


$$
\begin{aligned}
g= & -2 \nabla \Delta^{2} u \nabla \phi-\Delta^{2} u \Delta \phi \\
& -\Delta\left(u \Delta^{2} \phi+4 \nabla \Delta u \nabla \phi+6 \Delta u \Delta \phi+4 \nabla u \nabla \Delta \phi\right)-\phi \Delta\left(|u|^{p-1} u\right)
\end{aligned}
$$

The semigroup representation formula for $\omega$ gives that

$$
\omega(t)=e^{(t+1) \Delta^{3}} \omega(-1)+\int_{-1}^{t} e^{(t-s) \Delta^{3}} g(s) d s \quad \text { for } \quad-1 \leq t<0,
$$

where $e^{t \Delta^{3}}$ is the semigroup associated with the equation $u_{t}-\Delta^{3} u=0$ in $\mathbb{R}^{n}$, i.e.,

$$
\left(e^{t \Delta^{3}} h\right)(x)=\int_{\mathbb{R}^{n}} \Gamma(x-y, t) h(y) d y .
$$

Notice that $\int_{\mathbb{R}^{n}} \Gamma(x-y, t) d y=1$. It follows that

$$
\left\|e^{t \Delta^{3}} h\right\| \leq\|h\|_{\infty} \text {. }
$$

The (3.1) implies that

$$
\begin{aligned}
\left|\left(e^{t \Delta^{3}} D_{i} h\right)(x)\right| & =\left|\int_{\mathbb{R}^{n}} \Gamma(x-y, t) D_{i} h(y) d y\right| \\
& =\left|\int_{\mathbb{R}^{n}} \frac{\partial}{\partial x_{i}} \Gamma(x-y, t) h(y) d y\right| \leq C t^{-\frac{1}{6}}\|h\|_{\infty}, \quad \forall i=1,2, \ldots, n,
\end{aligned}
$$

So, we get that

$$
\begin{aligned}
& \left\|e^{t \Delta^{3}} D_{i} h\right\|_{\infty} \leq C t^{-\frac{1}{6}}\|h\|_{\infty}, \quad\left\|e^{t \Delta^{3}} D_{i j} h\right\|_{\infty} \leq C t^{-\frac{1}{3}}\|h\|_{\infty}, \\
& \left\|e^{t \Delta^{3}} D_{i j k} h\right\|_{\infty} \leq C t^{-\frac{1}{2}}\|h\|_{\infty}, \quad\left\|e^{t \Delta^{3}} D_{i j k m} h\right\|_{\infty} \leq C t^{-\frac{2}{3}}\|h\|_{\infty}, \\
& \left\|e^{t \Delta^{3}} D_{i j k m q} h\right\|_{\infty} \leq C t^{-\frac{5}{6}}\|h\|_{\infty},
\end{aligned}
$$

where $i, j, k, m, q \in\{1,2, \cdots, n\}$.

Now let $g=g_{1}+g_{2}$, where $g_{2}=-\phi \Delta\left(|u|^{p-1} u\right)$. As above, we estimate

$$
\begin{aligned}
& \left|\int_{-1}^{t} e^{(t-s) \Delta^{3}} g_{2}(s) d s\right| \\
\leq & \int_{-1}^{t}\left|\int_{\mathbb{R}^{n}} \Delta(\phi \Gamma(x-y, t-s))\left(|u|^{p-1} u\right)(y, s) d y\right| d s \\
\leq & \left.\int_{-1}^{t}\left|\int_{\mathbb{R}^{n}} \Delta \Gamma(x-y, t-s) \phi\right| u\right|^{p-1} u(y) d y \mid d s \\
& +\left.\int_{-1}^{t}\left|\int_{\mathbb{R}^{n}}(\Gamma(x-y, t-s) \Delta \phi+2 \nabla \Gamma(x-y, t-s) \cdot \nabla \phi)\right| u\right|^{p-1} u(y) d y \mid d s \\
& \leq C \int_{-1}^{t}(t-s)^{-\frac{1}{3}}\left\|\phi u^{p}\right\|_{\infty}(s) d s+C \int_{-1}^{t}\left\|\Delta \phi u^{p}\right\|_{\infty}(s) d s
\end{aligned}
$$




$$
\begin{aligned}
& +C \int_{-1}^{t}(t-s)^{-\frac{1}{6}}\left\|\nabla \phi u^{p}\right\|_{\infty}(s) d s \\
\leq & C \int_{-1}^{t}(t-s)^{-\frac{1}{3}}\|u\|_{\infty}^{p-1}\|\omega\|_{\infty}(s) d s+C \int_{-1}^{t}\left\|u^{p}\right\|_{\infty}(s) d s \\
& +C \int_{-1}^{t}(t-s)^{-\frac{1}{6}}\|u\|_{\infty}^{p}(s) d s \\
\leq & C \varepsilon^{p-1} \int_{-1}^{t}(t-s)^{-\frac{1}{3}}(-s)^{-\frac{2}{3}}\|\omega\|_{\infty}(s) d s+C \varepsilon^{p} \int_{-1}^{t}(-s)^{-\frac{2 p}{3(p-1)}} d s \\
& +C \varepsilon^{p} \int_{-1}^{t}(t-s)^{-\frac{1}{6}}(-s)^{-\frac{2 p}{3(p-1)}} d s,
\end{aligned}
$$

due to our assumption.

On the other hand, it is found similarly that

$$
\begin{aligned}
& \left|\int_{-1}^{t} e^{(t-s) \Delta^{3}} g_{1}(s) d s\right| \\
= & \mid \int_{-1}^{t} \int_{\mathbb{R}^{n}} \Gamma(x-y, t-s)\left(-2 \nabla \Delta^{2} u \nabla \phi-\Delta^{2} u \Delta \phi\right. \\
& \left.-\Delta\left(u \Delta^{2} \phi+4 \nabla \Delta u \nabla \phi+6 \Delta u \Delta \phi+4 \nabla u \nabla \Delta \phi\right)\right) d y d s \mid \\
& \leq C \int_{-1}^{t}(t-s)^{-\frac{5}{6}}\|u\|_{\infty}(s) d s \leq C \varepsilon \int_{-1}^{t}(t-s)^{-\frac{5}{6}}(-s)^{-\frac{2}{3(p-1)}} d s .
\end{aligned}
$$

By (3.2)-(3.4), (3.6) and (3.7), we get that for $-1 \leq t<0$,

$$
\begin{aligned}
\|\omega(t)\|_{\infty} \leq & \varepsilon+\varepsilon^{p-1} \int_{-1}^{t}(t-s)^{-\frac{1}{3}}(-s)^{-\frac{2}{3}}\|\omega\|_{\infty}(s) d s \\
& +C \varepsilon^{p} \int_{-1}^{t}(t-s)^{-\frac{1}{6}}(-s)^{-\frac{2 p}{3(p-1)}} d s+C \varepsilon \int_{-1}^{t}(t-s)^{-\frac{5}{6}}(-s)^{-\frac{2}{3(p-1)}} d s \\
\leq & \varepsilon+C \varepsilon^{p-1} \int_{-1}^{t}(t-s)^{-\frac{1}{3}}(-s)^{-\frac{2}{3}}\|\omega\|_{\infty}(s) d s+C \varepsilon(-t)^{\frac{1}{6}-\frac{2}{3(p-1)}},
\end{aligned}
$$

due to $1<p<3$ and Lemma (1).

Let $y(t)=\|\omega(t)\|_{\infty}$; therefore

$$
y(t) \leq \varepsilon+C \varepsilon(-t)^{\frac{1}{6}-\frac{2}{3(p-1)}}+C \varepsilon^{p-1} \int_{-1}^{t}(t-s)^{-\frac{1}{3}}(-s)^{-\frac{2}{3}} y(s) d s .
$$

Define $f(t)=\chi_{[-1,0]}(t) y(t), \forall t<0$. We introduce a special maximal function on $(-\infty, 0)$ :

$$
(M f)(t)=\sup _{r>0} \frac{1}{r} \int_{t-r}^{t}|f(s)| d s, \quad \forall t \in(-\infty, 0) .
$$


Now $\forall r>0$,

$$
\begin{aligned}
& \int_{-1}^{t}(t-s)^{-\frac{1}{3}}(-s)^{-\frac{2}{3}} y(s) d s=\int_{-\infty}^{t}(t-s)^{-\frac{1}{3}}(-s)^{-\frac{2}{3}} f(s) d s \\
& =\int_{t-r}^{t}(t-s)^{-\frac{1}{3}}(-s)^{-\frac{2}{3}} f(s) d s+\int_{-\infty}^{t-r}(t-s)^{-\frac{1}{3}}(-s)^{-\frac{2}{3}} f(s) d s \\
& =I_{1}+I_{2} .
\end{aligned}
$$

We compute these two integrals, respectively.

$$
\begin{aligned}
I_{1} & \leq(-t)^{-\frac{2}{3}} \int_{t-r}^{t}(t-s)^{-\frac{1}{3}} f(s) d s \\
& =(-t)^{-\frac{2}{3}} \sum_{k=0}^{\infty} \int_{t-\frac{r}{2^{k}}}^{t-\frac{r}{2^{k+1}}}(t-s)^{-\frac{1}{3}} f(s) d s \\
& \leq(-t)^{-\frac{2}{3}} \sum_{k=0}^{\infty}\left(\frac{r}{2^{k+1}}\right)^{-\frac{1}{3}} \int_{t-\frac{r}{2^{k}}}^{t-\frac{r}{2^{k+1}}} f(s) d s \\
& \leq(-t)^{-\frac{2}{3}} \sum_{k=0}^{\infty}\left(\frac{1}{2^{k+1}}\right)^{\frac{2}{3}} r^{\frac{2}{3}}(M f)(t) \\
& =C r^{\frac{2}{3}}(-t)^{-\frac{2}{3}}(M f)(t),
\end{aligned}
$$

and

$$
I_{2} \leq r^{-\frac{1}{3}} \int_{-\infty}^{t-r}(-s)^{-\frac{2}{3}} f(s) d s \leq r^{-\frac{1}{3}} \int_{-\infty}^{t}(-s)^{-\frac{2}{3}} f(s) d s=r^{-\frac{1}{3}} \int_{-1}^{t}(-s)^{-\frac{2}{3}} f(s) d s .
$$

Then,

$f(t) \leq \varepsilon+C \varepsilon(-t)^{\frac{1}{6}-\frac{2}{3(p-1)}}+C \varepsilon^{p-1}\left[r^{\frac{2}{3}}(-t)^{-\frac{2}{3}}(M f)(t)+r^{-\frac{1}{3}} \int_{-1}^{t}(-s)^{-\frac{2}{3}} f(s) d s\right]$,

for all $r>0$ and $t \in(-\infty, 0)$.

Let

$$
r=\frac{\int_{-1}^{t}(-s)^{-\frac{2}{3}} f(s) d s}{(-t)^{-\frac{2}{3}}(M f)(t)}
$$

so we have

$$
\begin{aligned}
f(t) \leq & \varepsilon+C \varepsilon(-t)^{\frac{1}{6}-\frac{2}{3(p-1)}}+C \varepsilon^{p-1}\left((-t)^{-\frac{1}{3}} \int_{-1}^{t}(-s)^{-\frac{2}{3}} f(s) d s\right)^{\frac{2}{3}}((M f)(t))^{\frac{1}{3}} \\
\leq & \varepsilon+C \varepsilon(-t)^{\frac{1}{6}-\frac{2}{3(p-1)}}+C \varepsilon^{p-1}(-t)^{-\frac{1}{3}} \int_{-1}^{t}(-s)^{-\frac{2}{3}} f(s) d s \\
& +C \varepsilon^{p-1}(M f)(t) .
\end{aligned}
$$


If we define

$$
g(t)=(-t)^{-\frac{1}{3}} \int_{-1}^{t}(-s)^{-\frac{2}{3}} f(s) d s,
$$

then

$$
g^{\prime}(t)=(-t)^{-1}\left[\frac{1}{3}(-t)^{-\frac{1}{3}} \int_{-1}^{t}(-s)^{-\frac{2}{3}} f(s)+f(t)\right] \geq 0 .
$$

Hence $g(t)$ is increasing in $(-\infty, 0)$.

Then we get

$$
\begin{aligned}
\max _{-1 \leq \tau \leq t} f(\tau) \leq & \varepsilon+C \varepsilon(-t)^{\frac{1}{6}-\frac{2}{3(p-1)}} \\
& +C \varepsilon^{p-1} g(t)+C \varepsilon^{p-1} \max _{-1 \leq \tau \leq t}(M f)(\tau), \quad \forall t \in[-1,0),
\end{aligned}
$$

where we have used $\frac{1}{6}-\frac{2}{3(p-1)}<0$ since $1<p<3$.

Clearly, $\max _{-1 \leq \tau \leq t}(M f)(\tau) \leq \max _{-1 \leq \tau \leq t} f(\tau)$ by our definition of the maximal function. Therefore (3.11) implies that for any $-1 \leq t<0$,

$$
\begin{gathered}
\max _{-1 \leq \tau \leq t} f(\tau) \leq \\
\frac{1}{1-C \varepsilon^{p-1}}\left[\varepsilon+C \varepsilon(-t)^{\frac{1}{6}-\frac{2}{3(p-1)}}+C \varepsilon^{p-1}(-t)^{-\frac{1}{3}} \int_{-1}^{t}(-s)^{-\frac{2}{3}} f(s) d s\right],
\end{gathered}
$$

provided that $C \varepsilon^{p-1}<1$. Especially,

$$
\begin{aligned}
f(t) \leq & \frac{1}{1-C \varepsilon^{p-1}}\left[\varepsilon+C \varepsilon(-t)^{\frac{1}{6}-\frac{2}{3(p-1)}}+C \varepsilon^{p-1}(-t)^{-\frac{1}{3}} \int_{-1}^{t}(-s)^{-\frac{2}{3}} f(s) d s\right] \\
& \forall t \in[-1,0) .
\end{aligned}
$$

Then for $\varepsilon>0$ small enough, we obtain

$$
\begin{aligned}
(-t)^{\frac{1}{3}} f(t) \leq & 2\left[\varepsilon+C \varepsilon(-t)^{\frac{1}{2}-\frac{2}{3(p-1)}}+C \varepsilon^{p-1} \int_{-1}^{t}(-s)^{-1}(-s)^{\frac{1}{3}} f(s) d s\right] \\
& \forall t \in[-1,0) .
\end{aligned}
$$

Define $h(t)=(-t)^{\frac{1}{3}} f(t)$; then

$$
h(t) \leq 2 \varepsilon+2 C \varepsilon(-t)^{\frac{1}{2}-\frac{2}{3(p-1)}}+2 C \varepsilon^{p-1} \int_{-1}^{t}(-s)^{-1} h(s) d s,
$$

Applying Lemma(2), we have

$$
\begin{aligned}
h(t) & \leq(-t)^{-2 C \varepsilon^{p-1}}\left[2 \varepsilon+C(p, \varepsilon) \varepsilon(-t)^{\frac{1}{2}-\frac{2}{3(p-1)}+2 C \varepsilon^{p-1}}\right] \\
& \leq 2 \varepsilon(-t)^{-2 C \varepsilon^{p-1}}+C(p, \varepsilon) \varepsilon(-t)^{\frac{1}{2}-\frac{2}{3(p-1)}}, \quad \forall t \in[-1,0) .
\end{aligned}
$$

Then $f(t) \leq 2 \varepsilon(-t)^{-\frac{1}{3}-2 C \varepsilon^{p-1}}+C(p, \varepsilon) \varepsilon(-t)^{\frac{1}{6}-\frac{2}{3(p-1)}}, \forall t \in[-1,0)$, or

$$
y(t) \leq 2 \varepsilon(-t)^{-\frac{1}{3}-2 C \varepsilon^{p-1}}+C(p, \varepsilon) \varepsilon(-t)^{\frac{1}{6}-\frac{2}{3(p-1)}}, \quad \forall t \in[-1,0) .
$$


Choose $\varepsilon>0$ small enough that $\frac{1}{3}+2 C \varepsilon^{p-1}<\frac{2}{3(p-1)}$ which is possible since $1<p<3$. Define $\alpha=\max \left\{\frac{1}{3}+2 C \varepsilon^{p-1}, \frac{2}{3(p-1)}-\frac{1}{6}\right\} \leq \frac{2}{3(p-1)}$, it is easy to find that $\alpha>\frac{1}{3}$; then (3.13) implies $y(t) \leq C(p, \varepsilon) \varepsilon(-t)^{-\alpha}, \forall t \in[-1,0)$. Hence

$$
|u(x, t)| \leq C(p, \varepsilon) \varepsilon(-t)^{-\alpha}, \quad \forall(x, t) \in B_{\frac{1}{2}}(0) \times[-1,0) .
$$

Now let $\tilde{\phi}$ be a function supported on $B_{\frac{1}{2}}(o)$ with $\tilde{\phi} \equiv 1$ on $B_{\frac{1}{4}}(0)$ and $0 \leq \tilde{\phi} \leq 1$, and define $\tilde{\omega}=\tilde{\phi} u$; then we go back to (3.6)-(3.8) and we have that

$$
\begin{aligned}
\|\tilde{\omega}(t)\|_{\infty} \leq & \varepsilon+C \int_{-1}^{t}(t-s)^{-\frac{1}{3}}\|u\|_{\infty}^{p-1}\|\tilde{\omega}\|_{\infty} d s+C \int_{-1}^{t}\|u\|_{\infty}^{p} d s \\
& +C \int_{-1}^{t}(t-s)^{-\frac{1}{6}}\|u\|_{\infty}^{p} d s+C \varepsilon \int_{-1}^{t}(t-s)^{-\frac{5}{6}}\|u\|_{\infty} d s \\
\leq & \varepsilon+C \varepsilon^{p-1} \int_{-1}^{t}(t-s)^{-\frac{1}{3}}(-s)^{-\alpha(p-1)}(-s)^{-\alpha} d s+C \varepsilon^{p} \int_{-1}^{t}(-s)^{-\alpha p} d s \\
& +C \varepsilon^{p} \int_{-1}^{t}(t-s)^{-\frac{1}{6}}(-s)^{-\alpha p} d s+C \varepsilon \int_{-1}^{t}(t-s)^{-\frac{5}{6}}(-s)^{-\alpha} d s \\
\leq & \varepsilon+C \varepsilon^{p-1} \int_{-1}^{t}(t-s)^{-\frac{1}{3}}(-s)^{-\alpha p} d s+C \varepsilon^{p} \int_{-1}^{t}(-s)^{-\alpha p} d s \\
& +C \varepsilon^{p} \int_{-1}^{t}(t-s)^{-\frac{1}{6}}(-s)^{-\alpha p} d s+C \varepsilon \int_{-1}^{t}(t-s)^{-\frac{5}{6}}(-s)^{-\alpha} d s
\end{aligned}
$$

due to (3.14).

Since $\frac{1}{3}<\alpha<\frac{2}{3(p-1)}$, we get

$$
\frac{5}{6}-\alpha p>\frac{2}{3}-\alpha p>\frac{1}{6}-\alpha .
$$

Hence by Lemma(1), we obtain

$$
\|\tilde{\omega}(t)\|_{\infty} \leq \varepsilon+C \varepsilon^{p-1}+C \varepsilon^{p-1}(-t)^{\frac{1}{6}-\alpha} \leq\left(2+C \varepsilon^{p-1}\right)(-t)^{\frac{1}{6}-\alpha}, \quad \forall t \in[-1,0),
$$

Which means, for small $\varepsilon>0$,

$$
|u(x, t)| \leq\left(2+C \varepsilon^{p-1}\right)(-t)^{\frac{1}{6}-\alpha}, \quad \forall(x, t) \in B_{r}(0) \times[-1,0) .
$$

Iterating the argument finitely many times we can get that there is a number $0<$ $r_{0}<\frac{1}{4}$ such that

$$
|u(x, t)| \leq K(-t)^{-\frac{1}{6 p}}, \quad \forall(x, t) \in B_{r_{0}}(0) \times[-1,0),
$$

where $\mathrm{K}$ is constant. 
Next, we choose another cut-off function $\hat{\phi}$ supported on $B_{r_{0}}$ such that $\hat{\phi} \equiv 1$ on $B \frac{r_{0}}{2}$ and define $\hat{\omega}=\hat{\phi} u$. Going back to (3.15) and applying Lemma(1), we have

$$
\begin{aligned}
\|\hat{\omega}(t)\|_{\infty} \leq & \varepsilon+C \int_{-1}^{t}(t-s)^{-\frac{1}{3}}\|u\|_{\infty}^{p-1}\|\hat{\omega}\|_{\infty} d s+C \int_{-1}^{t}\|u\|_{\infty}^{p} d s \\
& +C \int_{-1}^{t}(t-s)^{-\frac{1}{6}}\|u\|_{\infty}^{p} d s+C \varepsilon \int_{-1}^{t}(t-s)^{-\frac{5}{6}}\|u\|_{\infty} d s \\
\leq & \varepsilon+C K^{p-1} \int_{-1}^{t}(t-s)^{-\frac{1}{3}}(-s)^{-\frac{1}{6}} d s+C K^{p} \int_{-1}^{t}(-s)^{-\frac{1}{6}} d s \\
& +C K^{p} \int_{-1}^{t}(t-s)^{-\frac{1}{6}}(-s)^{-\frac{1}{6 p}} d s+C K \int_{-1}^{t}(t-s)^{-\frac{5}{6}}(-s)^{-\frac{1}{6 p}} d s \\
\leq & +C K^{p-1}
\end{aligned}
$$

which means that $|u(x, t)| \leq C$ in $B \frac{r_{0}}{2} \times[-1,0)$. This completes the proof of the theorem.

Using the same argument, we can easily draw the following conclusion.

Theorem 3. Suppose $p \geq 3$, then for any $\delta \in\left(0, \frac{2}{3(p-1)}\right)$, there is a constant $\varepsilon>0$, depending on $n, p$ and the constant in (3.1), such that if $u$ is a solution of the equation

$$
u_{t}-\Delta\left(\Delta^{2} u-|u|^{p-1} u\right)=0, \quad \text { on } \quad Q_{r}=B_{r}(a) \times\left[t_{1}-r^{6}, t_{1}\right)
$$

where $a \in \mathbb{R}^{n}, t_{1} \in \mathbb{R}$ and $0<r \leq 1$, and if

$$
|u(x, t)| \leq \varepsilon\left(t_{1}-t\right)^{-\frac{2}{3(p-1)}} \quad \text { for } \text { all } \quad(x, t) \in Q_{r},
$$

then $u$ does not blow up at $\left(a, t_{1}\right)$.

\section{REFERENCES}

[1] J. W. Barrett, S. Langdon, and R. Nürnberg, "Finite element approximation of a sixth order nonlinear degenerate parabolic equation," Numer. Math., vol. 96, no. 3, pp. 401-434, 2004.

[2] T. Cheng and G.-F. Zheng, "On the blow-up of solutions for some fourth order parabolic equations," Nonlinear Anal., Theory Methods Appl., vol. 66, no. 11, pp. A, 2500-2511, 2007.

[3] C. M. Elliott and S. Zheng, "On the Cahn-Hilliard equation," Arch. Ration. Mech. Anal., vol. 96, pp. 339-357, 1986.

[4] J. D. Evans, V. A. Galaktionov, and J. R. King, "Unstable sixth-order thin film equation. I: Blow-up similarity solutions," Nonlinearity, vol. 20, no. 8, pp. 1799-1841, 2007.

[5] J. D. Evans, V. A. Galaktionov, and J. R. King, "Unstable sixth-order thin film equation. II: Global similarity patterns," Nonlinearity, vol. 20, no. 8, pp. 1843-1881, 2007.

[6] Y. Giga and R. V. Kohn, "Nondegeneracy of blow up for semilinear heat equations," Commun. Pure Appl. Math., vol. 42, no. 6, pp. 845-884, 1989.

[7] A. Jüngel and J.-P. Milišić, "A sixth-order nonlinear parabolic equation for quantum systems," SIAM J. Math. Anal., vol. 41, no. 4, pp. 1472-1490, 2009. 
[8] V. A. Solonnikov, "On boundary value problems for linear parabolic systems of differential equations of general form,” Proc. Steklov Inst. Math., vol. 83, p. 184, 1965.

\section{Authors' addresses}

\section{Zhenbang Li}

Department of Mathematics, Jilin University, Changchun 130012, China

E-mail address: jamesbom23@yahoo.com.cn

\section{Changchun Liu}

Department of Mathematics, Jilin University, Changchun 130012, China

E-mail address: liuccejlu.edu.cn 